\title{
Investigación tecnológica aplicada: Domocaña
}

\author{
Applied technology research: Domocaña
}

\author{
$\underline{\text { R. Barrionuevo }}^{(*)}$
}

RESUMEN

En Perú, hay una creciente necesidad de vivienda, en especial para las familias de extrema pobreza; además de ocurrencia de sismos y deterioro del medio ambiente. Es por esto que se busca nuevas soluciones con calidad, accesibles y ecológicas.

Estudios realizados en Perú y otros países han mostrado la bondad de la caña o bambú como estructura sismo resistente, de distribución, cerramiento o decorativa. Sin embargo, su uso para vivienda de interés social es limitado; la población la usa en forma precaria.

En ese contexto, se propuso y se desarrolló el techo Domocaña en la Facultad de Arquitectura de la Universidad Nacional de Ingeniería (UNI). Fueron satisfactorios los ensayos de cargas estáticas en el Laboratorio del Centro de Investigaciones Sísmicas y Mitigación de Desastres (CISMID-UNI). Las viviendas experimentales que se construyeron utilizando la tecnología mejorada del adobe y el techo domocaña resistieron cargas dinámicas en los sismos de Moquegua (2003) y Pisco (2007) sin sufrir daño alguno.

La UNI, por encargo de la Comisión del Ministerio de Vivienda, Construcción y Saneamiento del Perú, y con el fin de dar la norma técnica, está estudiando las características físicas y mecánicas del material para establecer parámetros de diseño y condiciones constructivas para el uso de la caña y bambú en edificaciones.

113.100

Palabras clave: domocaña, cúpula de caña, techo ecológico, techo de bambú, techo económico.

\section{SUMMARY}

In Peru, there is a growing need for housing, especially for families in extreme poverty; as well as occurrence of earthquakes and environmental degradation. That is the reason why new solutions with quality, accessible and ecological are searched.

Studies in Peru and other countries have shown the cane or bamboo goodness as structure earthquake resistant, distribution, enclosure or decorative. However, its use for social housing is limited; population uses it in a precarious way.

In this context, the Domocaña Roof was proposed by the Architecture School of the National Engineering (UNI). Trials of static charges carried out in the Laboratory of Seismic Research Centre and Disaster Mitigation (CISMID-UNI) were satisfactory. The experimental houses built using the improved technology of adobe and Domocaña roof resisted dynamic loads in the earthquakes of Moquegua (2003) and Pisco (2007) without suffering any damage.

UNI, by order of the Commission of the Ministry of Housing, Construction and Sanitation of Peru, and to give technical standard, is studying the physical and mechanical properties of the material to establish design parameters and construction conditions for the use of cane and bamboo buildings.

Keywords: domocaña dome cane, green roof, bamboo ceiling, roof ecobambú.
Informes de la Construcción Vol. 63, 523, 51-58, julio-septiembre 2011 ISSN: 0020-0883 eISSN: 1988-3234 doi: 10.3989/ic.10.025 

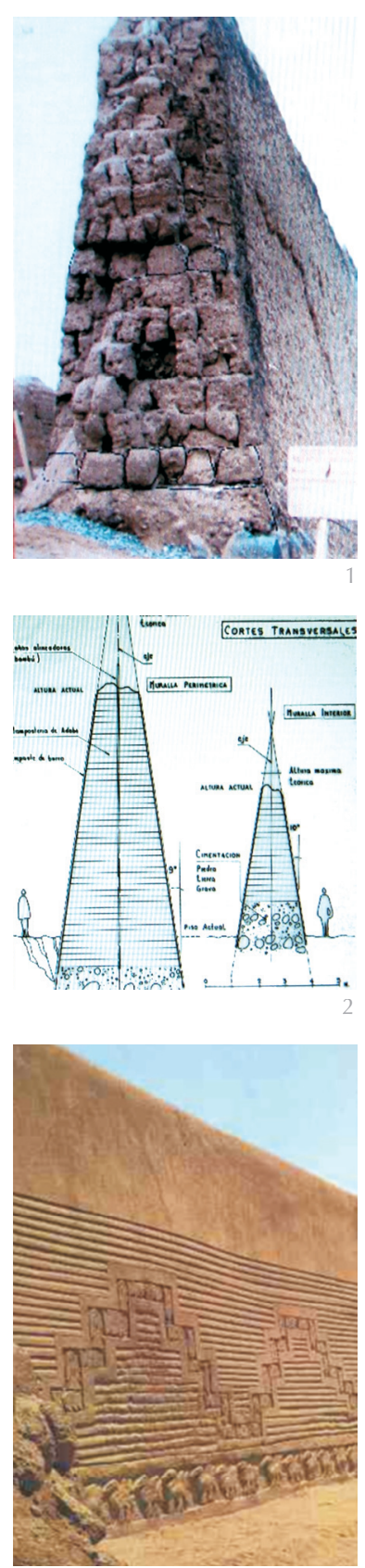

1-3. Museo de Chan Chan, Perú

\section{LA MOTIVACIÓN}

En el Perú, al igual de otros países en vías de desarrollo, existe la "urgente necesidad de atender la cada vez más creciente demanda de vivienda", en especial la de los marginados, sector mayoritario en el cual es más agudo el problema y que por su extrema pobreza, no tiene acceso a programas de gobierno.

Los esfuerzos realizados por los programas de gobierno no están al alcance de los que están en extrema pobreza. No se atiende ni siquiera al $10 \%$ del déficit actual (2.200.000 viviendas (1), que se incrementa en 90.000 cada año). La iniciativa popular sigue "resolviendo" el problema, construyendo con esfuerzo un espacio donde vivir, con graves deficiencia técnicas y de seguridad. Al principio en forma incipiente, utilizando esteras, madera o tierra, ya sea en la ciudad o en el campo.

Este problema se acrecienta por las características climáticas y ecológicas existentes en el Perú, distintas en toda su extensión, ocurrencia frecuente de sismos, muchas veces severos, vientos fuertes, aluviones, sequías, Iluvias torrenciales, entre otros desastres; diferentes culturas, así como problemas sociales y económicos; realidad que incide fuertemente en la tarea de construir en el Perú y en las tecnologías a utilizar.

Por otro lado, Perú cuenta con abundantes recursos naturales que pueden ser utilizados para abordar el problema. Tradicionalmente las tecnologías utilizadas son el concreto armado y las albañilerías en zona urbana, y el adobe y la madera en zona rural. La formación de los profesionales arquitectos e ingenieros civiles incide en el uso de materiales y procedimientos constructivos mencionados, sobre los cuales existen normas técnicas. Las empresas industriales y constructoras, así como las instituciones financieras orientan sus actividades para esa realidad.

Los investigadores peruanos han desarrollado o tienen en proceso investigaciones tecnológicas aplicadas a la vivienda y a la construcción, con resultados exitosos y se han desarrollado proyectos experimentales, didácticos y demostrativos de construcción de viviendas.

Tabla 1

Características estructurales de diferentes materiales ${ }^{2}$

\begin{tabular}{|c|c|c|c|c|c|}
\hline Material & $\begin{array}{c}\text { Resistencia } \\
\text { de diseño } \\
\mathbf{K g} / \mathbf{~ c m}^{\mathbf{2}}\end{array}$ & $\begin{array}{c}\text { Masa por } \\
\text { Volumen } \\
\mathbf{K g} / \mathbf{~ m}^{\mathbf{3}}\end{array}$ & $\begin{array}{c}\text { Relación } \\
\text { Resist./Masa } \\
\mathbf{R} / \mathbf{M}\end{array}$ & $\begin{array}{c}\text { Módulo de } \\
\text { Elasticidad } \\
\mathbf{K g} / \mathbf{~ c m}^{\mathbf{2}}\end{array}$ & $\begin{array}{c}\text { Relación de } \\
\text { Elast / Rigid. } \\
\mathbf{E} / \mathbf{~ M ~}\end{array}$ \\
\hline Hormigón & 82 & 2.400 & 0,032 & 127.400 & 53 \\
\hline Acero & 1.630 & 7.800 & 0,209 & $2^{\prime} 40.000$ & 274 \\
\hline Madera & 76 & 600 & 0,127 & 112.000 & 187 \\
\hline Bambú & 102 & 600 & 0,127 & 112.000 & 340 \\
\hline
\end{tabular}

\section{LA VIVIENDA SUSTENTABLE}

Según PNUMA (2), "la construcción sustentable es el conjunto de actividades orientadas a satisfacer las necesidades de vivienda, su entorno y la infraestructura del presente sin comprometer la capacidad de dar respuestas a las demandas de las generaciones futuras". Ese concepto obliga no sólo a pensar en el creciente déficit de vivienda, sino también en soluciones que no agredan el medio ambiente. Todos los actores involucrados en esa tarea, los gobiernos, la actividad privada, diseñadores y constructores, organismos no gubernamentales e inclusive la comunidad organizada deben tomar conciencia de ello.

Hay experiencia acumulada, investigaciones tecnológicas aplicadas a la vivienda y la construcción, tanto urbana como rural, que involucran no sólo materiales y tecnologías, sino también el diseño arquitectónico, incorporando aspectos ecológicos, bioclimáticos y socioculturales. Los resultados de aplicación, a pequeña escala, son exitosos pero quedan sólo en lo anecdótico porque no hay una aplicación masiva. Hay barreras que vencer y es el reto que debe ser asumido.

En ese contexto, en la Universidad Nacional de Ingeniería (UNI), se escogió un recurso natural renovable: LA CAÑA, por sus características mecánicas y físicas, las ventajosas posibilidades que significan su uso en la construcción de viviendas y por su capacidad de reducir el $\mathrm{CO}_{2}$. Se presentan los resultados alcanzados y las principales aplicaciones experimentales realizadas en los ámbitos nacional e internacional, así como la aceptación alcanzada por parte de la población interesada.

\section{EL ESTADO DEL ARTE}

Desde épocas muy antiguas, el mayor desarrollo de aplicación de las cañas en la construcción, sobretodo la especie conocida como bambú, se ha dado en Asia, desde la India a la China (3) y de Japón a Java. Por constituir una alternativa ante materiales más costosos, más de mil millones de personas habitan en viviendas construidas con caña o bambú. En América ha alcanzado notables avances en Colombia, Ecuador y Costa Rica, principalmente, pero aún no se han Ilegado a normas técnicas de diseño y construcción. En la Tabla $n^{\circ} 1$ se presenta una comparación sobre las características del hormigón, el acero, la madera y el bambú.

Son pocos los diseñadores estructurales de edificaciones de bambú y sólo se basan en experiencias prácticas. En el Perú, la caña se usó desde la época precolombina. En el museo de sitio de Chan Chan (Figuras $n^{\circ} 1$ al 3) se 
Tabla 2

Reforzamiento de muros de adobe

\begin{tabular}{|c|l|}
\hline Esbeltez (h / e) & \multicolumn{1}{c|}{ REFUERZOS INTERNOS } \\
\hline$\lambda=\mathrm{h} / \mathrm{e}$ & $\begin{array}{l}\text { Refuerzos de caña: } \\
\text { Rh + Rv : no se requiere }\end{array}$ \\
\hline $\boldsymbol{\lambda} \leq \mathbf{6}$ & $\begin{array}{l}\text { Refuerzos de caña: } \\
\text { Rh + Rv : sólo en encuentro de muros }\end{array}$ \\
\hline $\mathbf{6} \leq \boldsymbol{\lambda} \leq \mathbf{8}$ & $\begin{array}{l}\text { Refuerzos de caña: } \\
\text { Rh + Rv se requiere en toda la longitud de los muros }\end{array}$ \\
\hline
\end{tabular}

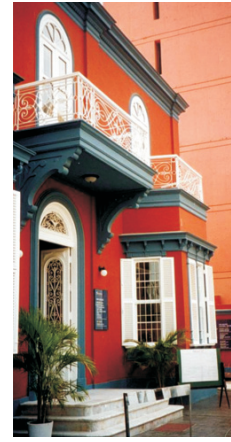

4. Vivienda en Lima

5. Vivienda antigua en Moquegua

6. Carrizo

7. Caña de guayaquil

8. Caña brava

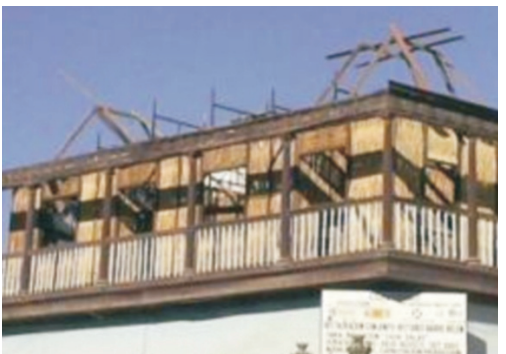

aprecian muros de adobe con caña, pero sin función estructural sino para el alineamiento del muro. En Caral se ha encontrado también vestigios del uso de cañas en sus muros.

En la época del virreinato se construyeron viviendas (Figura 4) y templos con adobe en el primer piso y quincha en el segundo piso, que hasta ahora perduran.

Edificaciones de quincha en el centro histórico de la ciudad peruana de Moquegua de más de trescientos años (Figura 5), fueron dañadas en el sismo del año 2000 y están en proceso de rehabilitación. El recubrimiento de los muros ha sido retirado y se puede apreciar que la caña está en un $90 \%$ en perfecto estado.

\section{LA CAÑA EN PERÚ}

Las cañas son gramíneas de gran avidez, crecen cerca a fuentes de agua y tienen un rápido crecimiento. Son abundantes en regiones tropicales y subtropicales, templadas e inclusive a $4.000 \mathrm{~m}$ sobre el nivel del mar, si las condiciones ecológicas sean apropiadas. Están en todos los continentes, con excepción de Europa. Existen más de mil quinientas especies en el mundo. Su crecimiento es espontáneo, pero hay países en los que se promueve su cultivo.

En el Perú existen diferentes especies, pero aún su uso es limitado en la construcción. Se puede planificar su cultivo para aprovechar sus cualidades y para tener un abastecimiento suficiente, gracias a su rápido crecimiento. Las principales especies más utilizadas en el Perú (se da su nombre científico) son las siguientes: carrizo (chusquea spp), la caña bra- va (gynesium sagitatum) y la caña de guayaquil o bambú (guadua angustifolia).(4)

\section{INVESTIGACIONES MÁS IMPORTANTES EN PERÚ}

\subsection{Refuerzo estructural de muros de adobe}

La Universidad Nacional de Ingeniería (UNI), el Instituto Nacional de investigación y Normalización de la Vivienda (ININVI) Y la Pontificia Universidad Católica del Perú (PUCP) realizaron sendas investigaciones, cuyos resultados mostraron que la caña era un excelente refuerzo para los muros de adobe, dándole así características sismorresistentes, dentro de ciertas condiciones. Estos estudios permitieron llegar a la Norma Técnica $N^{\circ}$ 080: ADOBE, en la cual se establecen, entre otras, las características que

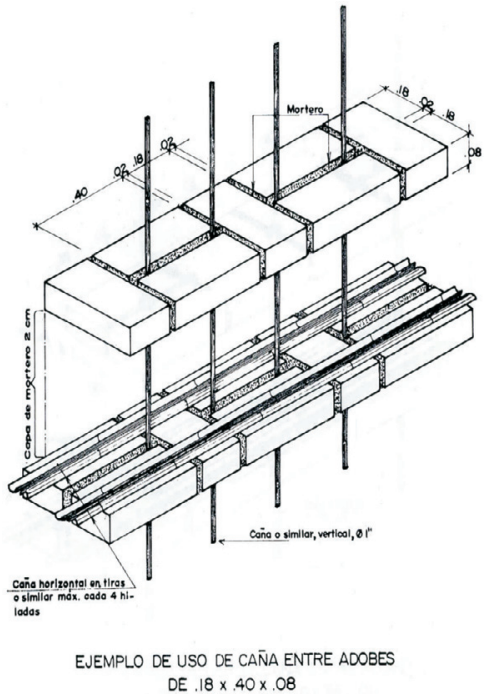




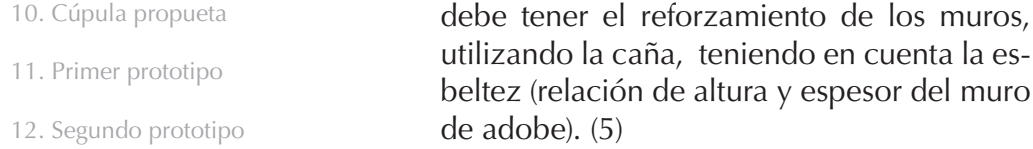

debe tener el reforzamiento de los muros, utilizando la caña, teniendo en cuenta la esbeltez (relación de altura y espesor del muro de adobe). (5)
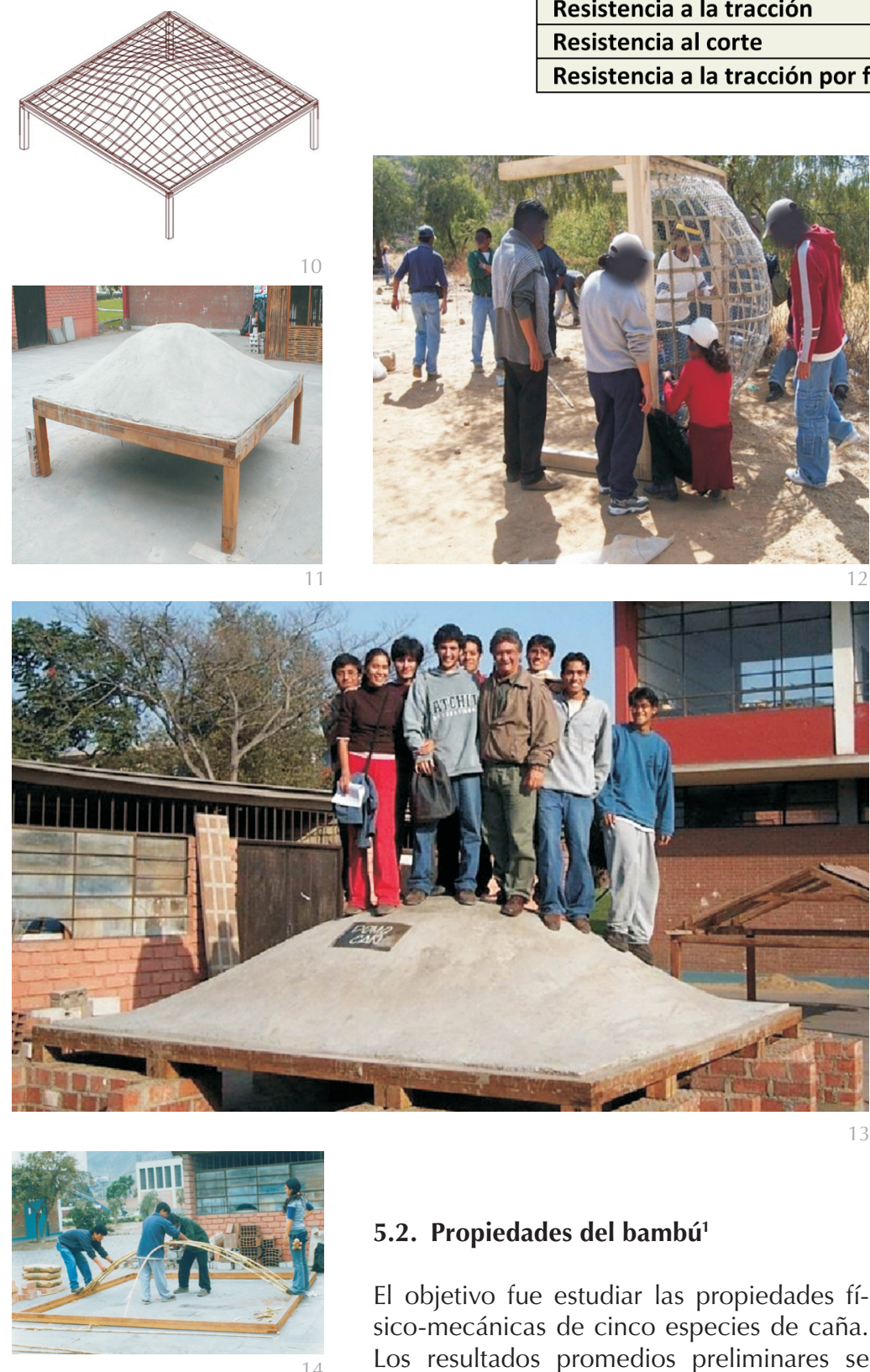

1 Jefe del estudio: Ing. Isabel Moromi

2 Jefe del estudio: Ing. Raquel Barrionuevo de Machicao. Premio a prototipos de innovación tecnológica otorgado por CONCYTEC.

${ }^{3}$ Centro de Investigación Sísmica y de Mitigación de Desastres

\subsection{Propiedades del bambú ${ }^{1}$}

El objetivo fue estudiar las propiedades físico-mecánicas de cinco especies de caña. Los resultados promedios preliminares se muestran en la Tabla $n^{\circ}$ 3. (6)

Los valores dependen de la especie, pero se presenta una gran variabilidad entre especimenes de una misma especie, por lo que es necesario evaluar la influencia de otros factores, como: edad, grado de madurez, defectos y condiciones ambientales en que se han desarrollado. El contenido de humedad de los especimenes varió entre $8 \%$ y $15 \%$ y la densidad entre 0,28 y 0,84 . Sin embargo, según el avance realizado permite conside- rar que los valores de resistencia obtenidos son suficientes para los esfuerzos que normalmente se presentan en las viviendas de interés social y edificaciones similares.

Tabla 3

Valores de resistencia de la caña ensayada

\begin{tabular}{|l|l|}
\hline Resistencia a la compresión & varía entre 226 a $559 \mathrm{~kg} / \mathrm{cm} 2$ \\
\hline Resistencia a la tracción & varía entre 154 a $862 \mathrm{~kg} / \mathrm{cm} 2$ \\
\hline Resistencia al corte & varía entre 74 a $146 \mathrm{~kg} / \mathrm{cm} 2$ \\
\hline Resistencia a la tracción por flexión & varía entre 543 a $746 \mathrm{~kg} / \mathrm{cm} 2$ \\
\hline
\end{tabular}

5.3. El Domocaña ${ }^{2}$

La búsqueda de un techo económico que utilice recursos locales, de fácil construcción y sismorresistente llevó a proponer el uso de la caña (carrizo, caña brava o bambú), como material constructivo por sus excelentes características de resistencia y por ser un material conocido y usado en la fabricación de esteras (lámina de caña trenzada muy usada en el Perú). Se usó malla gallinero, revestimiento de mortero cemento-arena o barro, con características de seguridad, habitabilidad y bajo costo.

La investigación se orientó también a lograr un proceso constructivo de fácil aprendizaje para ser aplicado por la comunidad beneficiaria o por pequeñas microempresas. Con ese fin se fabricaron dos prototipos para estudiar y validar la propuesta constructiva; uno con estudiantes y el otro con miembros de una comunidad marginada. El resultado fue satisfactorio por lo racional y simple, fácilmente comprendido por quienes, alumnos y pobladores, nunca habían construido.

Otros dos prototipos se construyeron para verificar su comportamiento estructural ante cargas estáticas de servicio. Los ensayos en el CISMID ${ }^{3}$ - UNI, comprobaron que funciona bien y que, probablemente, su respuesta también sería adecuada ante cargas sísmicas, principalmente debido a su capacidad para incursionar en el intervalo no lineal.

La metodología empleada comprendió los siguientes pasos:

- Verificación del estado del arte con relación a las actuales soluciones de techos para vivienda de bajo costo.

- Verificación del uso de las cañas en el Perú y en el mundo.

- Análisis teórico para definir la forma más adecuada y verificar el comportamiento estructural del techo propuesto

- Construcción de prototipos para estudiar el proceso constructivo y analizar los costos resultantes.

- Ensayos técnicos de cargas estáticas para conocer el comportamiento es- 
tructural del techo. Análisis del resultado.

- Verificación del comportamiento del domocaña ante cargas dinámicas.

- Validación del proceso constructivo en construcciones experimentales para conocer si su proceso es fácilmente entendido y realizado por la población.

\subsection{Descripción de la investigación}

\section{a. La forma}

Los estudios teóricos revisados y realizados muestran que la losa plana trabaja a tracción y a compresión, en cambio la cúpula en todos sus puntos trabaja a la compresión, con valores más bajos cuanto más alta es la flecha central. (7). Por lo tanto, aprovechando la flexibilidad de las cañas, se adoptó la forma de doble curvatura o cúpula, dejando que tomen su propia forma. Además, estéticamente el resultado obtenido fue un espacio arquitectónicamente agradable, amplio y cálido.

Esta característica permite tener muros relativamente bajos, más estables y más seguros, lo que incide en un menor costo. Una ventaja adicional del Domocaña es que reparte la carga sobre cuatro muros que lo sustentan, amarrándolos y mejorando su comportamiento ante cargas laterales.

\section{b. Los prototipos}

El primer prototipo se construyó con carrizo de $25 \mathrm{~mm}$ de diámetro, formando una retícula; malla gallinero con cocada de $12 \mathrm{~mm}$, colocada en tres capas, dos sobre la parte superior de la retícula de caña y una en su parte inferior para contribuir a la rigidez y facilitar la adherencia del recubrimiento.

El segundo prototipo se construyó para validar el proceso constructivo, con los miembros de una comunidad de escasos recursos, ubicada en la zona marginal de la ciudad de Cochabamba, en Bolivia. Las características fueron las mismas del primer prototipo, usando cañas típicas del lugar. La facilidad del proceso constructivo permitió construir el módulo en un día y medio, incluidos los marcos de confinamiento; se comprobó que puede ser construido aun por personas que nunca han intervenido en tareas similares.

El tercer y cuarto prototipos se construyeron para verificar el comportamiento del Domocaña ante la solicitación de cargas estáticas. Los prototipos fueron de 3,40 m x 3,40 m y con una flecha de 1,00 m.

Una primera prueba fue someter el tercer prototipo al peso de 10 personas (750 kilos) que se subieron progresivamente sobre su superficie superior, con un peso total de 750 $k$. El resultado fue muy satisfactorio porque no sufrió ninguna deformación.

Según la Norma E 020 Cargas del Reglamento Nacional de Edificaciones (RNE) del Perú, las cúpulas se diseñan para una carga viva repartida de $50 \mathrm{~kg} / \mathrm{m}^{2}$. Para los ensayos del tercer y cuarto prototipos (el de bambú y el de carrizo) se decidió someterlos a una carga viva de 5 veces ese valor, es decir 250 $\mathrm{kg} / \mathrm{cm}^{2}$, con un total de 2.880 kilos.

Se definieron 5 puntos sobre la superficie del tercer prototipo para registrar las alturas con relación a la base, y así definir exactamente la forma inicial y la deformación final que adopta el Domocaña durante el proceso de carga progresiva. Esos mismos puntos, se usaron para verificar la recuperación de la forma del domo, retirando también las cargas progresivamente. Se programaron cinco oportunidades de medición: antes de colocar las cargas, inmediatamente después de colocarlas, un día antes de retirarlas, inmediatamente después de retirarlas y 5 días después de retirar las cargas. Los resultados se aprecian en el Tabla $\mathrm{n}^{\circ} 4$

Tabla 4

Deformaciones registradas

\begin{tabular}{|c|c|c|c|c|c|}
\hline $\begin{array}{r}\text { Lectura } \\
\text { puntos }\end{array}$ & A1 & A4 & C6 & A7 & A5 \\
\hline 1 & 0,00 & 0,00 & 0,00 & 1,00 & 0,00 \\
\hline 2 & 1,50 & 2,00 & 1,40 & 2,00 & 1,50 \\
\hline 3 & 1,90 & 2,50 & 2,30 & $\mathbf{2 , 5 5}$ & 2,50 \\
\hline 4 & 1,10 & 1,00 & 1,30 & 1,50 & 0,00 \\
\hline 5 & 0,80 & 1,00 & 0,70 & 1,00 & 0.70 \\
\hline
\end{tabular}

El ensayo del cuarto prototipo (de bambú) fue más riguroso. Se construyó en forma similar al de caña brava, la única diferencia fue que la superficie superior de la retícula de bambú enmallada fue revestida con mortero de cemento - arena, en proporción 1:2. Se ubicó a 1,00 m de altura del piso, para facilitar las lecturas. Los diales fueron ubicados debajo del domo en los cinco puntos previamente definidos.

La superficie interior quedó cara vista. La retícula de bambú se armó en el piso y luego se colocó en el marco metálico de pruebas. Fue necesario asegurar los bastidores de madera

Tabla 5

Pesos por lectura realizada

\begin{tabular}{|l|l|c|}
\hline 1a lectura & Cargas del 1 al 10 & 400 kilos \\
\hline 2a lectura & Cargas del 11 al 16 & 240 kilos \\
\hline 3a lectura & Cargas del 17 al 24 & 320 kilos \\
\hline 4a lectura & Cargas del 25 al 32 & 320 kilos \\
\hline 5a lectura & Cargas del 33 al 40 & 320 kilos \\
\hline 6a lectura & Cargas del 41 al 52 & 480 kilos \\
\hline 7a lectura & Cargas del 53 al 72 & 800 kilos \\
\hline \multicolumn{2}{|c|}{ TOTAL CARGAS } & 2.880 KILOS \\
\hline
\end{tabular}

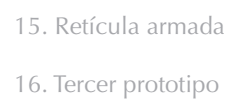

17. Prototipo enmarallado

18. Tercer prototipo terminado

\section{Dial de lectura}

20. Domocaña con cargas de servicio
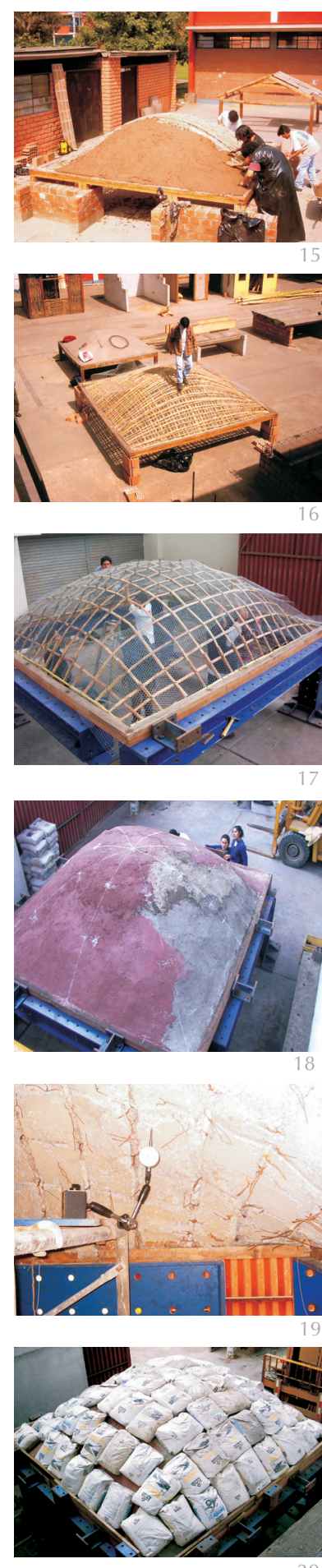
Tabla 6

Lecturas de las cargas realizadas y deflexiones producidas LECTURAS REALIZADAS

\begin{tabular}{|c|c|c|c|c|c|c|c|}
\hline \multirow{2}{*}{$\begin{array}{c}\text { No } \\
\text { Lectura }\end{array}$} & \multirow{2}{*}{ Tiempo } & \multirow{2}{*}{$\begin{array}{c}\text { Cargas } \\
\text { (kilos) }\end{array}$} & \multicolumn{5}{|c|}{ DEFLEXIONES / puntos } \\
\cline { 4 - 8 } & & $\mathrm{cm}$ & $\mathrm{cm}$ & $\mathrm{cm}$ & 7 & $\mathrm{~cm}$ & $\mathrm{~cm}$ \\
\cline { 4 - 8 }
\end{tabular}

*19-agosto

\begin{tabular}{|l|c|c|c|c|c|c|c|}
\hline 1 & 10,08 & 400 & 0,000 & 0,095 & 0,000 & 0,157 & 0,330 \\
\hline 2 & 10,27 & 240 & 0,000 & 0,170 & 0,000 & 0,205 & 0,363 \\
\hline 3 & 10,54 & 320 & 0,087 & 0,270 & 0,000 & 0,362 & 0,490 \\
\hline 4 & 11,05 & 320 & 0,225 & 0,378 & 0,000 & 0,587 & 0,610 \\
\hline 5 & 11,16 & 320 & 0,350 & 0,480 & 0,000 & 0,656 & 0,715 \\
\hline 6 & 11,45 & 480 & 0,535 & 0,620 & 0,000 & 0,940 & 0,871 \\
\hline 7 & 12,37 & 800 & 0,936 & 0,930 & 0,095 & 1,385 & 1,332 \\
\hline 8 & 17,50 & 2.880 & 0,980 & 1,040 & 0,095 & 1,410 & 1,375 \\
\hline
\end{tabular}

*20-agosto-03

\begin{tabular}{|l|l|l|l|l|l|l|l|}
\hline $\mathbf{9}$ & 8,40 & 2.880 & 1,450 & 1,080 & 0,095 & 1,440 & 1,440 \\
\hline
\end{tabular}

*20-agosto-03

\begin{tabular}{|l|c|c|c|c|c|c|c|}
\hline 11 & 14,35 & 2.880 & 1,090 & 1,140 & 0,0860 & 1,420 & 1,468 \\
\hline 12 & 15,30 & -800 & 0,900 & 0,966 & 0,0710 & 1,265 & 1,360 \\
\hline 13 & 15,45 & -480 & 0,800 & 0,885 & 0,0628 & 1,126 & 1,238 \\
\hline 14 & 16,00 & -320 & 0,730 & 0,831 & 0,0570 & 1,052 & 1,188 \\
\hline 15 & 16,04 & -320 & 0,630 & 0,735 & 0,0478 & 0,917 & 1,070 \\
\hline 16 & 16,05 & -320 & 0,500 & 0,727 & 0,0370 & 0,750 & 0,945 \\
\hline 17 & 16,07 & -240 & 0,410 & 0,727 & 0,0310 & 0,660 & 0,860 \\
\hline 18 & 16,16 & -400 & 0,234 & 0,727 & 0,0130 & 0,416 & 0,700 \\
\hline 19 & 16,36 & 0 & 0,036 & 0,727 & 0,0109 & 0,328 & 0,629 \\
\hline
\end{tabular}

21. Diagrama cargas vs,

deformación

22. Vivienda con techo

Domocaña
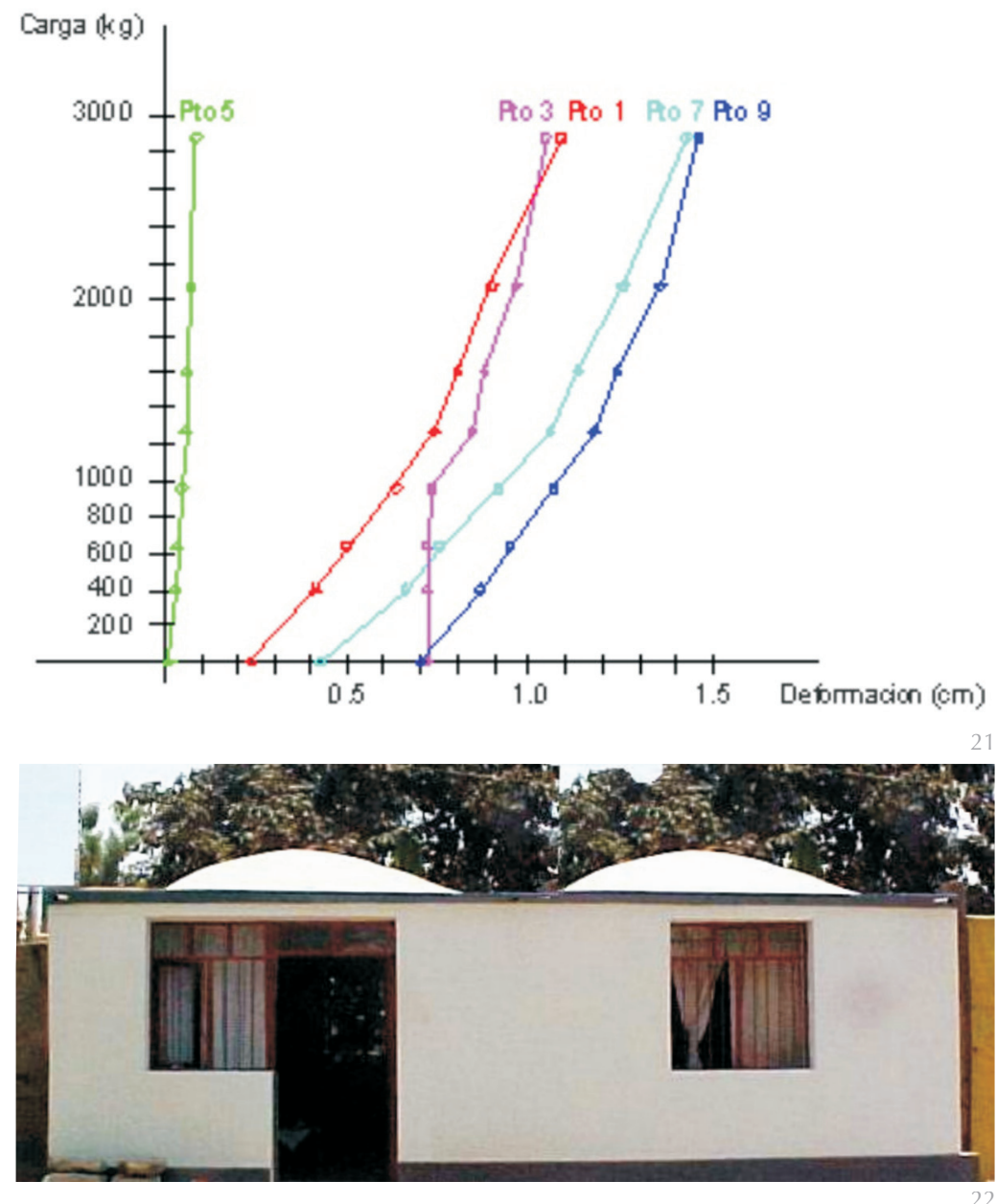

al marco metálico de prueba para evitar su desplazamiento durante el ensayo.

Para el ensayo del domocaña de bambú se definieron 5 puntos sobre su superficie, con el fin de registrar las deflexiones producidas por las cargas, y luego verificar la recuperación de la forma del domo, después de retirar las cargas.

Se usaron instrumentos de precisión, con lecturas cada vez que se colocaban las cargas, desde 0 kilos hasta alcanzar un total de 2.880 kilos. Los resultados de los ensayos se indican en la tabla 6 y Figura 21. En los 5 puntos, ubicados sobre la superficie, se realizaron las lecturas sucesivas de la deformación que se fue produciendo mientras se colocaban progresivamente las cargas.

En la tabla $\mathrm{n}^{\circ} 6$ se presentan las lecturas programadas y los pesos que se fueron colocando en cada oportunidad.

Sería bueno obtener gráficas de los Domocaña en ensayos de cargas sísmicas para ver su comportamiento inelástico, aunque la verificación de los domocaña construidos en viviendas de Moquegua e Ica, donde ocurrieron fuertes sismos es ya un avance en ese sentido porque mostraron una respuesta adecuada ante cargas sísmicas, principalmente debido a su capacidad para incursionar en el intervalo no lineal.

Los resultados hasta ahora alcanzados muestran que el Domocaña es una alternativa para viviendas económicas, por sus condiciones de seguridad, calidad, costo, bajo peso, uso de un recurso renovable y su simple proceso constructivo.

\subsection{Revestimiento del Domocaña (4)}

Se pueden usar varios tipos de revoques en base a diferentes materiales, desde los más económicos como el barro mezclado con paja, hasta los más sofisticados. Se debe tener siempre en cuenta factores tan importantes como la adherencia, durabilidad, resistencia al impacto, a las lluvias y otros factores que inciden en la habitabilidad.

Por información recogida en el estudio de las construcciones tradicionales de quincha antigua peruana (alrededor de 300 años), en el que se constató su buen estado de conservación; así como en la experiencia lograda en los ensayos y aplicaciones experimentales del ININVI y otras instituciones, los siguientes tipos de revestimiento se pueden aplicar al Domocaña:

- Revestimiento primario de barro y paja, en una proporción de 2,0 kg de paja por $100 \mathrm{~kg}$ de tierra, enrasado con el marco del Domoca- 
ña. Revestimiento secundario, aplicado sobre el revestimiento primario seco, de $1,5 \mathrm{~cm}$ o 2,0 cm de espesor, de barro preparado con tierra areno arcillosa cernida, con un impermeabilizante, como el asfalto RC-250 (aproximadamente un $3 \%$ en peso). Un revestimiento final de "lechada" de cemento aplicada con brocha a modo de pintura. La superficie usualmente debe pintarse dos veces.

- Un revestimiento primario de suelo-cemento enrasado con el marco del domocaña. Utilizar tierra arenosa y un porcentaje de cemento según el tipo de tierra, que puede variar de $7 \%$ a $10 \%$ en volumen. Este revestimiento deberá ser curado, por lo menos una semana, mediante humedecimiento periódico. Un revestimiento final de $1,5 \mathrm{~cm}$ a 2,0 cm de mortero cemento-cal-arena en la proporción en volumen 1:1:5. En zonas Iluviosas se usará algún impermeabilizante.

- Un revestimiento primario igual que el de revoque Tipo 2 . Al final un revestimiento de $1,5 \mathrm{~cm}$ a $2,0 \mathrm{~cm}$ con un mortero preparado mezclado cemento-arena fina en la propor-
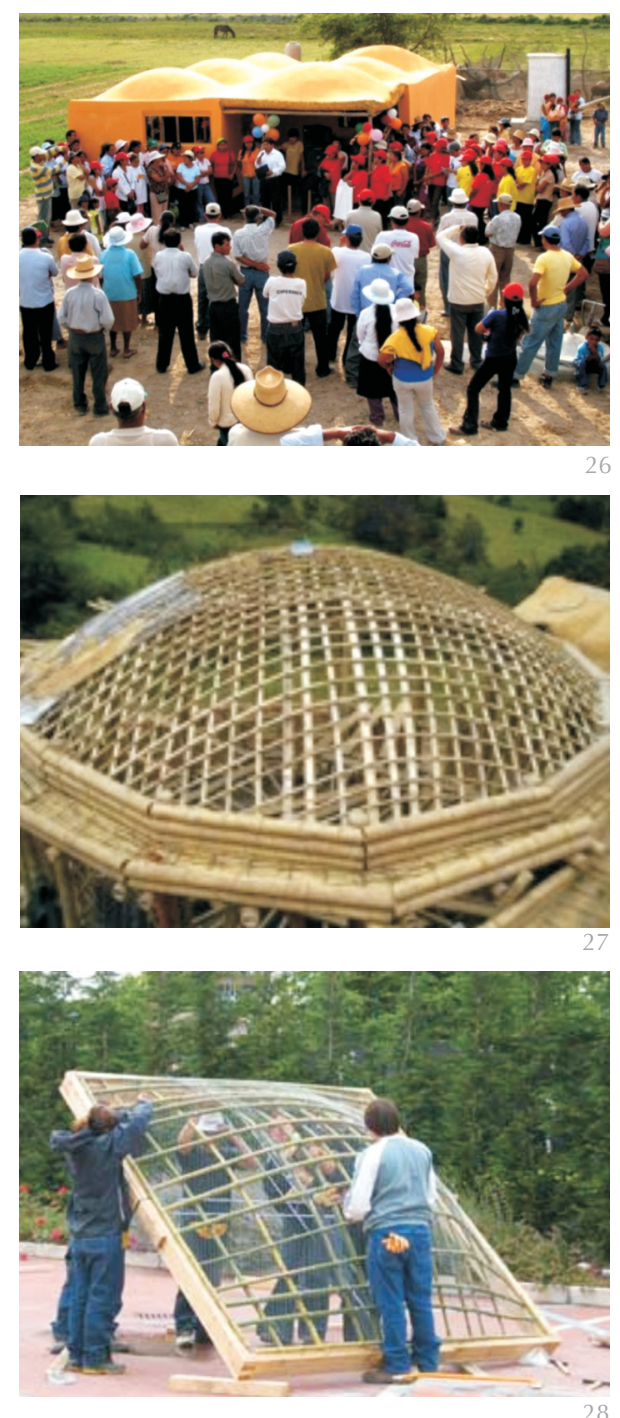

ción en volumen 1:5. En zonas lluviosas se usará algún impermeabilizante.

\section{APLICACIONES EXPERIMENTALES}

\section{Vivienda en Moquegua}

Como aplicación experimental se llevó a cabo un programa de construcción de viviendas con tecnologías nativas mejoradas en la ciudad de Moquegua, al sur del Perú, para transferir a los beneficiarios los conocimientos a través de la modalidad de capacitación-acción. Una de las tecnologías seleccionadas fue la tecnología mejorada del adobe con el techo Domocaña. Ante un sismo de $5,8^{\circ}$, su comportamiento fue satisfactorio, sin daño alguno, mientras que las casas de adobe vecinas fueron seriamente afectadas.
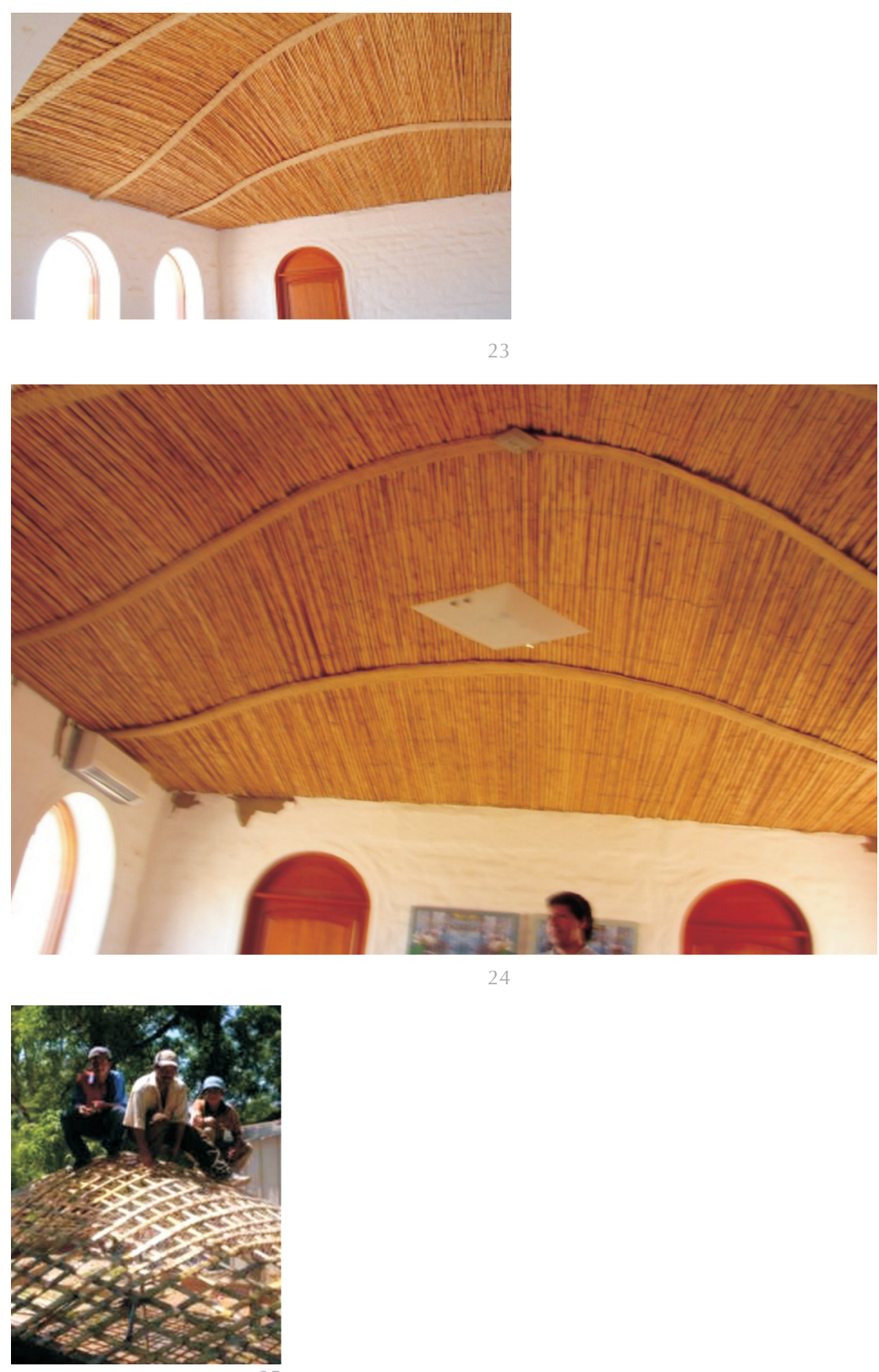

23. Vivienda de Humay antes del sismo

24. Vivienda de Humay después del sismo

26. Primera vivienda en Cabeza de Toro

27. Colombia

28. Milán
25. San Salvador 


\section{Vivienda en Humay}

Se construyó en la ciudad de Humay, Ica, una vivienda de adobe, con un ambiente techado con un domocaña de acabado cara vista, de $6 \times 6 \mathrm{~m}$.

El 15-8-2007 un fuerte sismo de 7,9 $9^{\circ}$ (epicentro en Pisco, muy cerca de Humay), puso en evidencia el buen comportamiento del Domocaña, mientras que el $70 \%$ de las viviendas de adobe existentes colapsaron.

Cabeza de Toro es una comunidad rural que sufrió graves daños en sus viviendas de adobe por efecto del sismo de Agosto de 2007, muchas de las cuales colapsaron. Una ONG peruana "Paz \& Esperanza" está realizando un programa de capacitación - acción para construir viviendas y ha adoptado las tecnologías de muros de estera y techo Domocaña. La comunidad ha asimilado rápidamente las tecnologías y actualmente ya se han edificado 157 viviendas.

Se presenta algunos de los ejemplos replicados y adoptados en distintos países.

\section{CONCLUSIONES}

- Los resultados alcanzados en la investigación del Domocaña son muy satisfactorios y confirman la hipótesis planteada.

- El procedimiento constructivo propuesto es muy simple, aún para personas que nunca realizaron tareas de construcción, lo que lo hace recomendable para la autoconstrucción.

- Es posible promover la formación de microempresarios para su producción, afirmación que se deriva del interés manifestado por quienes participaron en la investigación y sus aplicaciones experimentales. Indudablemente generará nuevas fuentes de trabajo.

- Los costos alcanzados por los prototipos construidos son muy competitivos con relación a otro tipo de techos, por los mínimos componentes que se requieren y su bajo costo, en zonas donde abunda el material. Su producción masiva bajaría aún más su costo.

- El tiempo que requiere para la construcción de un Domocaña es muy reducido, lo que incide favorablemente en menores costos.

- La forma de cúpula reduce permite reducir la altura de los muros, sin causar sensación de espacio reducido, da mayor estabilidad a los muros y, por lo tanto, el costo final de la edificación se reduce. Esa forma no tiene nada que envidiar a otros techos conocidos como "material noble". Es gratamente aceptada por los usuarios y quienes tuvieron la oportunidad de visitar los ambientes construidos con el Domocaña (profesionales, funcionarios, pobladores y otros).

- Los ensayos de cargas estáticas realizados, con valores más altos que los que establece la norma y el comportamiento de los 4 techos construidos en la ciudad de Moquegua, que soportó un sismo de 5,8 (escala de Ritcher), así como el construido en Humay, sometido a un fuerte sismo de 7,9 (epicentro en Pisco, muy cerca de Humay), mientras que el $70 \%$ de las viviendas de adobe existentes colapsaron, muestran que estructuralmente el techo funciona satisfactoriamente.

- La calidad estructural y las condiciones de habitabilidad de los ambientes techados con Domocañas, su bajo costo y la simplicidad de su construcción lo muestran como una alternativa de solución para viviendas de bajo costo accesibles a familias de escasos recursos.

- El uso de un recurso renovable y la promoción de su cultivo contribuye a la protección del medio ambiente.

En resumen, el Domocaña se puede aplicar ventajosamente en la construcción de viviendas y otras edificaciones por sus buenas condiciones de calidad, seguridad y costo.

\section{BIBLIOGRAFÍA}

(1) Informe del Banco Mundial (2009).

(2) Ornellas, A.: "Holcim, tras la construcción sustentable". Swissinfo.ch

(3) Hidalgo, O.: Bambú, el regalo de los dioses, pp. 448 - 472. Ed. Bamboscar2@007mundo.com, Bogotá.

(4) ININVI. La quincha prefabricada. pp. 8-10. Ediciones ININVI, Lima.

(5) Norma Técnica Nº 080: ADOBE del Reglamento Nacional de Edificaciones.

(6) Moromi, I. N.: Informe de la investigación de Propiedades del bambú.

(7) Lorenzo, P.; Massuh, H.; Leiva, L.; Barrionuevo, R. y otros: Cúpula de Ferrocemento. Ficha técnica en libro "Un techo para vivir", pp. 254-257. Ediciones UPC, Barcelona. 